\title{
Plasma antioxidants from chocolate
}

\section{Dark chocolate may offer its consumers health benefits the milk variety cannot match.}

here is some speculation that dietary flavonoids from chocolate, in particular (-)epicatechin, may promote cardiovascular health as a result of direct antioxidant effects or through antithrombotic mechanisms ${ }^{1-3}$. Here we show that consumption of plain, dark chocolate (Fig. 1) results in an increase in both the total antioxidant capacity and the (-)epicatechin content of blood plasma, but that these effects are markedly reduced when the chocolate is consumed with milk or if milk is incorporated as milk chocolate. Our findings indicate that milk may interfere with the absorption of antioxidants from chocolate in vivo and may therefore negate the potential health benefits that can be derived from eating moderate amounts of dark chocolate.

To determine the antioxidant content of different chocolate varieties, we took dark chocolateand milk chocolate prepared from the same batch of cocoa beans and defatted them twice with $n$-hexane before extracting them with a mixture of water, acetone and acetic acid (70.0:29.8:0.2 by volume). We measured their in vitro total antioxidant capacities using the ferric-reducing antioxidant potential (FRAP) assay ${ }^{4}$; FRAP values were $147.4 \pm 4.5$ and $78.3 \pm 3.4 \mu \mathrm{mol}$
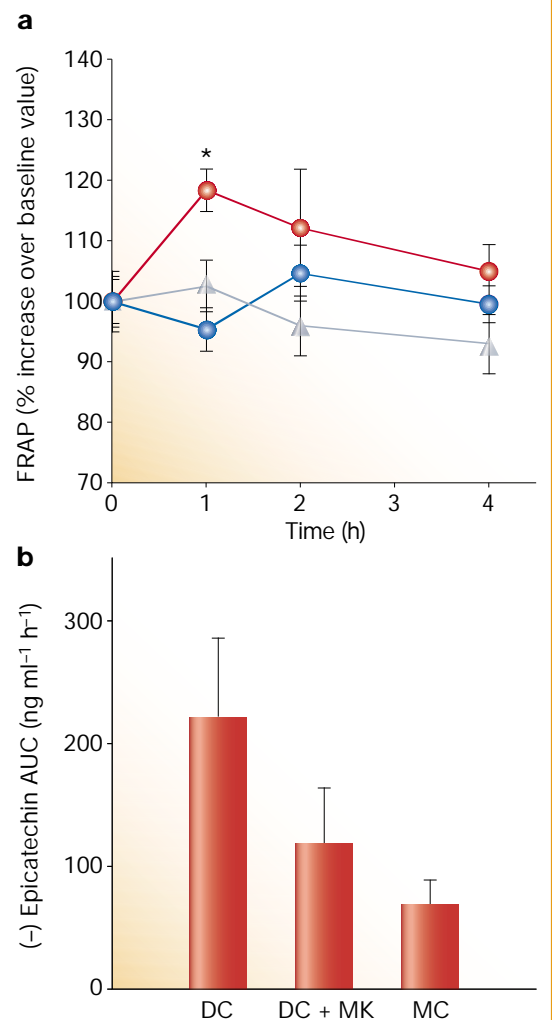

reduced iron per $100 \mathrm{~g}$ for dark and milk chocolate, respectively. Volunteers must therefore consume twice as much milk chocolate as dark chocolate to receive a similar intake of antioxidants.

We recruited 12 healthy volunteers ( 7 women and 5 men with an average age of $32.2 \pm 1.0$ years (range, $25-35$ years). Subjects were non-smokers, had normal blood lipid levels, were taking no drugs or vitamin supplements, and had an average weight of $65.8 \pm 3.1 \mathrm{~kg}$ (range, 46.0-86.0 kg) and body-mass index of $21.9 \pm 0.4 \mathrm{~kg} \mathrm{~m}^{-2}$ (range, 18.6-23.6 $\mathrm{kgm}^{-2}$ ). On different days, following a crossover experimental design, subjects consumed $100 \mathrm{~g}$ dark chocolate, $100 \mathrm{~g}$ dark chocolate with $200 \mathrm{ml}$ full-fat milk, or $200 \mathrm{~g}$ milk chocolate (containing the equivalent of up to $40 \mathrm{ml}$ milk).

One hour after subjects had ingested the chocolate, or chocolate and milk, we measured the total antioxidant capacity of their plasma by FRAP assay. Plasma antioxidant levels increased significantly after consumption of dark chocolate alone, from $100 \pm 3.5 \%$ to $118.4 \pm 3.5 \%$ (t-test, $\mathrm{P}<0.001)$, returning to baseline values $(95.4 \pm 3.6 \%)$ after $4 \mathrm{~h}$ (Fig. 2a). There was no significant changein plasmaFRAP values over the same period after ingestion of milk chocolate alone or of dark chocolate with milk (Fig. 2a).

The areas under the curves of (-)epicatechin plasma levels plotted against time ${ }^{5}$ were measured over the same 4-h period after ingestion for the three different conditions. Absorption of (-)epicatechin into the bloodstream after ingestion of chocolate was significantly less when the chocolate was accompanied by milk ( $-46.4 \pm 4.1 \%$; analysis of variance (ANOVA), $\mathrm{P}<0.001$ ) or if the chocolate itself contained milk

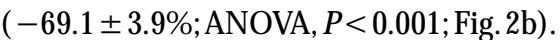

Addition of milk, either during ingestion or in the manufacturing process, therefore inhibits the in vivo antioxidant activity of chocolateand theabsorption into thebloodstream of (-)epicatechin. This inhibition

Figure 2 Effects of acute ingestion of $100 \mathrm{~g}$ dark chocolate (DC), $100 \mathrm{~g}$ dark chocolate with $200 \mathrm{ml}$ milk (DC + MK) or $200 \mathrm{~g}$ milk chocolate (MC) on the total antioxidant capacity (TAC) and (-)epicatechin content of human plasma. a, Mean TAC of plasma samples at the indicated times after chocolate consumption, expressed as ferric-reducing antioxidant potential (FRAP) ${ }^{4}$. Values are mean percentage increases $( \pm$ s.e.m.) relative to baseline values $(n=12)$. Red circles, DC; blue circles, $D C+M K$; grey triangles, MC. Asterisk denotes $P<0.001$. b, Mean $(-)$ epicatechin levels in plasma, expressed as the area under the curve in a (AUC, in $\mathrm{ng} \mathrm{ml}^{-1} \mathrm{~h}^{-1}$ ) for the 4-h period after chocolate consumption. Values are significantly different from one another (see text).

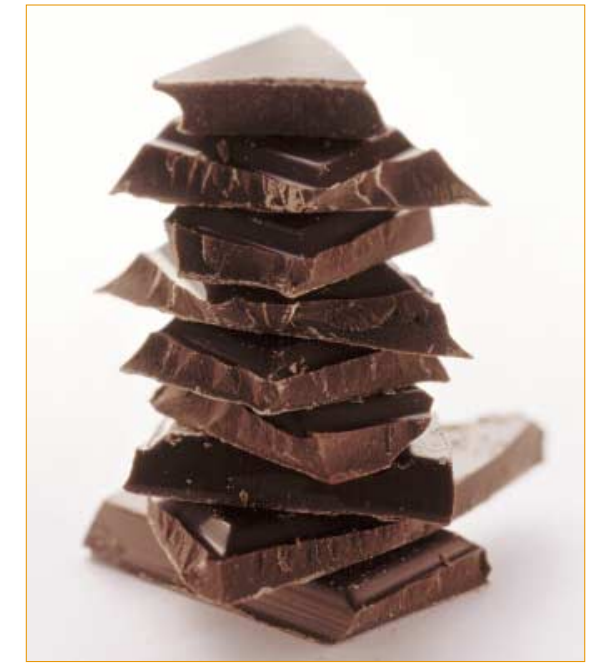

Figure 1 Stack of benefits? Unlike its milky counterpart, dark chocolate may provide more than just a treat for the tastebuds.

could be due to the formation of secondary bonds between chocolate flavonoids and milk proteins ${ }^{6,7}$, which would reduce the biological accessibility of the flavonoids and therefore the chocolate's potential antioxidant propertiesin vivo.

Our findings highlight the possibility that the in vivo antioxidant activity of flavonoids could be impaired by other dietary constituents. Other food combinations may also counteract the absorption and protective effects of flavonoids. There is therefore a need to take into account dietary habits when designing studies to assess the association between flavonoidrich foods, antioxidant activity and degenerativediseases.

\section{Mauro Serafini*, Rossana Bugianesi*,} Giuseppe Maiani*, Silvia Valtuena*, Simone De Santis*, Alan Croziert

*Antioxidant Research Laboratory, Unit of Human Nutrition, National Institute for Food and

Nutrition Research, Via Ardeatina 546,

00178 Rome, Italy

e-mail: serafini@inran.it

†Plant Products and Human Nutrition Group, Graham Kerr Building, Division of Biochemistry and M olecular Biology, Institute of Biomedical and Life Sciences, University of Glasgow,

Glasgow G12 8Q Q, UK

1. Rein, D. et al. J. Nutr. 130, 2109S-2114S (2000).

2. Holt, R. R., Schramm, D. D., Keen, C. L., Lazarus, S. A. \& Schmitz, H. H. J. Am. M ed. Assoc. 287, 2212-2213 (2002).

3. Steinberg, F. M., Bearden, M. N. \& Keen, C. L. J. Am. Diet. Assoc 103, 215-223 (2003).

4. Benzie, I. F. F. \& Strain, J. J. Anal. Biochem. 239, 229-240 (1996).

5. Maiani, G., Serafini, M., Salucci, M., Azzini, E. \& Ferro-Luzzi, A J. Chromatogr. B 692, 311-317 (1997).

6. Charlton, A. J. et al. J. Agric. Food Chem. 50, 1593-1601 (2002)

7. Serafini, M., Ghiselli, A. \& Ferro-Luzzi, A. Eur. J. Clin. Nutr. 50, 28-32 (1996).

Competing financial interests: declared none 